\title{
Combinations of modulators trained with the same and different target stimuli
}

\author{
ROBERT A. RESCORLA \\ University of Pennsylvania, Philadelphia, Pennsylvania
}

\begin{abstract}
In two autoshaping experiments, pigeons received training of several modulators with each of two target excitors. Experiment 1 used a conditioned-facilitation $(\mathbf{A}-\mathbf{X A}+)$ procedure, and Ex. periment 2 used a conditioned-inhibition $(A+/ X A-)$ procedure. In the first procedure, the $X$ modulators promoted responding to $A$, whereas in the second they depressed responding to $A$. Pairs of the $\mathrm{X}$ modulators were then presented in conjunction with a novel target stimulus, $B$. The ability of those pairs to modulate responding to $B$ was greater when the modulators in a pair had both been trained with the same $A$ stimulus rather than with different $A$ stimuli. That suggests an involvement of the original training targets in the transfer of modulators to a novel target.
\end{abstract}

A good deal of recent evidence suggests that some Pavlovian conditioning procedures endow a stimulus with the ability to modulate responding to signals of reinforcement (e.g., Holland, 1983, 1985; Rescorla, 1985). The two most frequently studied paradigms are facilitation (positive occasion-setting) and inhibition (negative occasionsetting). In the former, one stimulus (A) is followed by an unconditioned stimulus (US) only when $A$ is itself preceded or accompanied by another stimulus $(X)$. As a result of this $\mathrm{XA}+/ \mathrm{A}$ - treatment, responding to $A$ occurs primarily on trials also containing $X$. In the latter paradigm, the contingencies are reversed such that $\mathbf{A}$ is followed by the US only when it is not also preceded or accompanied by $\mathrm{X}$. Such an XA-/A+ paradigm constitutes the well-known conditioned-inhibition procedure in which responding to A occurs primarily on trials when it occurs alone. In many instances of such paradigms, $X$ appears to have gained the ability to modulate responding to $A$.

There has been considerable interest in how this modulation is accomplished. One can identify three principal loci for the action of $\mathrm{X}$ : on the activation of the A stimulus, of the US, or of the association between them. For instance, if $X$ were to affect the processing of $A$, it could reduce or amplify the response to $A$. Similarly, if $X$ were to govern the degree to which a representation of the US was available for activation, it might regulate the response to stimuli associated with the US. Finally, if $X$ were to gain control over the effective strength of the A-US association, it could modify the ability of $A$ to activate the US and so to evoke a response.

One approach to evaluating these possibilities has been to assess the degree to which X's modulatory power trans-

This research was supported by National Science Foundation Grants BNS 83-08176 and BNS 88-03514. Correspondence concerning this article should be addressed to Robert A. Rescorla, Department of Psychology, University of Pennsylvania, 3815 Walnut Street, Philadelphia, PA 19104. fers to stimuli other than the $A$ with which it was trained. For instance, a mode of operation in which $X$ governs the accessibility of the US representation anticipates that $X$ would modulate the response not only to $A$ but also to other stimuli associated with that US. However, an X that acted on either the processing of $A$ or the association between $A$ and the US should fail to transfer its modulation to alternative signals of the US. Considerable evidence supports the view that, at least in some preparations, an X can so transfer its effect to other stimuli, thus suggesting that at least a portion of its action is on the representation of the US (e.g., Holland, 1989a, 1989b, 1989c; Rescorla, 1985). However, the evidence also suggests that this transfer is often incomplete, indicating that some of X's action is somewhat specific to the stimulus with which it was trained.

One reason that X's action might show some specificity to the training $A$ is that $X$ undergoes perceptual modification when it is combined with various stimuli. As a result, $X$ should be slightly different, and hence less effective, when presented in conjunction with a transfer stimulus. Although it is difficult to rule out such perceptual modification, one recent report suggests that it fails to capture fully the tie of X to its particular A. Rescorla (1991) found that X's ability to transfer modulation from A to B was affected by changing the excitatory state of its $A$ prior to the time of transfer. In both inhibition and facilitation paradigms, adjusting the excitatory strength of $A$ had a specific effect on the ability of its $X$ to modulate responding to a transfer target $B$. Since such a manipulation should not have affected the degree to which $X$ undergoes perceptual change when transferred from $A$ to $B$, this implies that the specificity of $X$ to $A$ involves other processes.

The present paper reports additional evidence suggesting that the specificity of $X$ to $A$ goes beyond perceptual modification of $\mathrm{X}$ by $\mathrm{A}$. The intention was to study the involvement of the original target stimulus in transfer of a modulator by examining how different modulators com- 
bine their abilities. Several modulators were trained with either the same or different target stimuli and were then presented in compounds during transfer to another target stimulus. The question of interest was whether the success with which modulators combine during transfer will depend on the identities of the targets with which they were originally trained. If modulators act only to affect the accessibility of the US representation, then training with a common US should allow them to combine equally, whatever the identity of the particular target stimuli with which they were trained. On the other hand, if the targets themselves are involved in the transfer, then one might expect differentially successful combinations depending upon whether or not the component modulators shared a training target. Such dependence on shared or different training targets across compounds that are perceptually comparable would suggest an involvement of the targets other than by perceptual modification due to the context of presentation.

Experiment 1 used this logic within a conditionedinhibition paradigm. Experiment 2 used a parallel facilitation procedure. Both experiments used pigeon subjects in an autoshaping technique in which localized visual stimuli served as target stimuli, sometimes paired with a grain US. Such pairings result in directed pecking at the target stimuli. However, that pecking is readily modulated by the presence of auditory and visual stimuli in both inhibition and facilitation paradigms (e.g., Rescorla, 1985).

\section{EXPERIMENT 1}

This experiment assessed the transfer of conditioned inhibitors to a new excitor. Initially, the animals were trained with two excitatory keylights and four inhibitors. To minimize generalization among the inhibitors, two different types of stimuli were used, both of which have previously been shown to be successful inhibitors (e.g., Rescorla, 1985). Two of the inhibitors were diffuse auditory stimuli that began prior to the target and continued during its presence. Two of the inhibitors were keylights that immediately preceded the excitors but terminated with their onset. The inhibitors were organized in pairs so that one auditory and one visual inhibitor were used in conjunction with each of the two excitatory keylights. Each excitor was followed by grain when presented alone, but not when preceded by either of its inhibitors. Then, in a transfer test, all four possible auditory-visual combinations of inhibitors were presented in conjunction with a new excitor. The question of interest was whether the success of inhibitory transfer would depend on whether the two jointly presented inhibitors had been trained with the same or different targets.

\section{Method}

Subjects and Apparatus. The subjects were 16 female White Carneaux pigeons about 1 year old. They had participated in another autoshaping experiment that had used different stimuli and a different response key. The assignment of birds to groups in the present experiment was random with respect to their previous treatments. They were housed in pairs and maintained at $80 \%$ of their free-feeding weights.

The apparatus consisted of eight identical operant chambers, each measuring $27 \times 27 \times 35 \mathrm{~cm}$. The metal front panel of each chamber had a $5 \times 5 \mathrm{~cm}$ food magazine in its center, located $5 \mathrm{~cm}$ above the wire-mesh floor. Three response keys, $2.5 \mathrm{~cm}$ in diameter, were located one directly above the hopper and one on either side of the center of the front wall, $20 \mathrm{~cm}$ above the floor. Located behind the right key was an IEE in-line projector that permitted the transillumination of the key with five light stimuli: one blue (B), one yellow (Y), one white $(W)$, and two grid patterns of 1-mm black lines on a white background, one made of lines spaced $2 \mathrm{~mm}$ apart and oriented $45^{\circ}$ from the vertical and the other made of lines spaced $1 \mathrm{~mm}$ apart and oriented $-45^{\circ}$ from the vertical. The colored lights were confined to the bottom portion of the response key; the grid patterns and $W$ were confined to the top portion of the key. The other half of the key remained unilluminated in each case. These stimuli were generated by Ektachrome slides of drawings composed of Color-aid artist's paper.

The other three walls and the ceiling of the chambers were composed of clear Plexiglas. These chambers were placed in soundand light-attenuating shells, with ventilation fans providing background noise of $62 \mathrm{~dB}$ re $20 \mu \mathrm{N} / \mathrm{m}^{2}$. On the rear wall of each of those shells was mounted a 6-W bulb that was continuously illuminated during the session, except during the operation of the food hopper. The hopper contained Purina pigeon grain. Also mounted on that wall were two speakers that allowed the presentation of a white noise (N) and an $1800-\mathrm{Hz}$ tone $(\mathrm{T})$, each of which raised the sound level to about $80 \mathrm{~dB}$.

Experimental events were automatically controlled by relay equipment and microprocessors located in an adjoining room.

Procedure. Because of the birds' past experience in an autoshaping experiment, no magazine training was necessary. On the first day, they received a pretest of the visual stimuli. During that session, they received 12 presentations each of the two colors and the two grid patterns, each 5 sec long. During this and all subsequent sessions, the intertrial interval (ITI) was variable around a mean of $60 \mathrm{sec}$.

On each of the next 22 days, the birds received training intended to establish each of the grid patterns as an excitor and each of the colors and auditory stimuli as inhibitors. Each session contained 125 -sec presentations of each grid pattern, each followed by 5 sec of access to grain. In addition, each session contained 16 nonreinforced presentations of each pattern, 8 of which were accompanied by an auditory stimulus and 8 of which were accompanied by a color. For each bird, a particular auditory stimulus and color consistently accompanied a particular pattern, but the assignments were balanced across subjects. The auditory stimuli were $15 \mathrm{sec}$ long, and the grid patterns occupied the final $5 \mathrm{sec}$. The color inhibitors were $5 \mathrm{sec}$ long and immediately preceded the 5-sec grids. These different parameters for the different types of inhibitors have routinely been found to be successful in prior experiments on conditioned inhibition (e.g., Rescorla, 1985, 1989). Other experiments in our laboratory have found that overlap with the excitor improves the inhibitory control of the less salient, diffuse auditory stimuli; however, such overlap between visual inhibitors and the target sometimes produces substantial disruption of responding to the target even in the absence of inhibitory training.

Over the next 4 days, $W$ was prepared as a transfer target. On each day, the birds received 365 -sec presentations of $W$. On the first 2 days, all trials were followed by $5 \mathrm{sec}$ of grain; on the next 2 days, all trials were nonreinforced. Other work in this laboratory had suggested that such prior training and extinction make keylights especially sensitive as transfer targets. Over the next 3 days, the birds received three additional conditioned-inhibition training sessions, identical to those given before.

On the next day, all birds received an initial test session. This session began with 12 reinforced 5-sec presentations of the target 


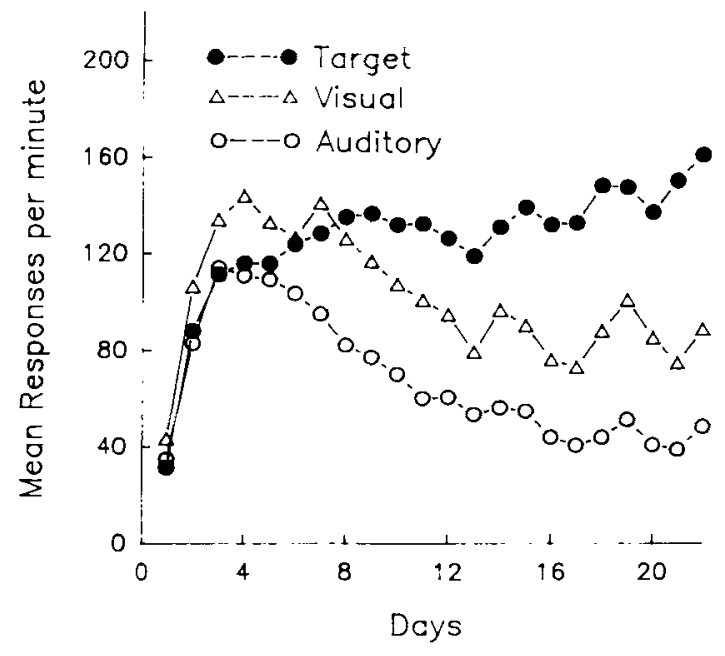

Figure 1. Responding to the target stimulus during acquisition of conditioned inhibition in Experiment 1. The keylight was reinforced when presented alone (target) but not when accompanied by auditory or visual inhibitors.

stimulus, $W$. It continued with 2 nonreinforced presentations each of $W$ alone and $W$ accompanied by each of the four possible combinations of auditory and visual inhibitors. The order of presentations was counterbalanced across animals with regard to the various types of compound and element trials. On compound trials, the inhibitors retained the same durations and temporal relations to the target as had been used during training. This test provides a comparison of the differential effectiveness of various combinations of inhibitors.

The birds then received a single day of retraining with the conditioned inhibitors, followed by a second transfer test day. That second test session was identical to the first except that intermingled with the two nonreinforced presentations of $W$ were eight nonreinforced inhibition trials, one with each of the individual inhibitors and one with each of the compounds. This test allows for an additional comparison of the inhibitory power of each type of compound with that of the elements, when each is transferred to a common target.

\section{Results and Discussion}

Figure 1 shows the course of acquisition of inhibition. Because there were no reliable differences as a function of stimulus identity, the results have been combined for the two excitors and within each inhibitory stimulus modality. Figure 1 shows responding during the excitors (targets) when they are presented alone and in combination with either visual or auditory inhibitors.

The course of acquisition is characteristic of that typically observed in this preparation. Responding rose rapidly during the excitor on all three kinds of trials, but with continued training, responding to the excitor declined when it was accompanied by either a visual or an auditory inhibitor. By the end of training, responding was reliably lower on both visual trials $[T(16)=4, p<.01]$ and auditory trials $[T(16)=0, p<.01]$. As might have been expected on the basis of previous reports, the auditory stimuli were somewhat more successful as inhibitors $[T(16)=1, p<.01]$. It is not clear whether this results from the difference in modality or in temporal relation to the excitor. The target stimulus, $W$, rapidly acquired responding, but then lost it with extinction. Because that training somewhat disrupted the inhibitors, three additional training sessions were given to restore their level of control.

The results from the first test session with various auditory and visual compound inhibitors are shown in Figure 2. That figure shows the mean rate of responding during $W$ when it was presented alone and when it was presented in compound with pairs of inhibitors that had been trained with either the same or a different target excitor. As a result of the retraining of $W$ at the beginning of the test session, it evoked substantial responding when it was presented alone. This responding was reduced by

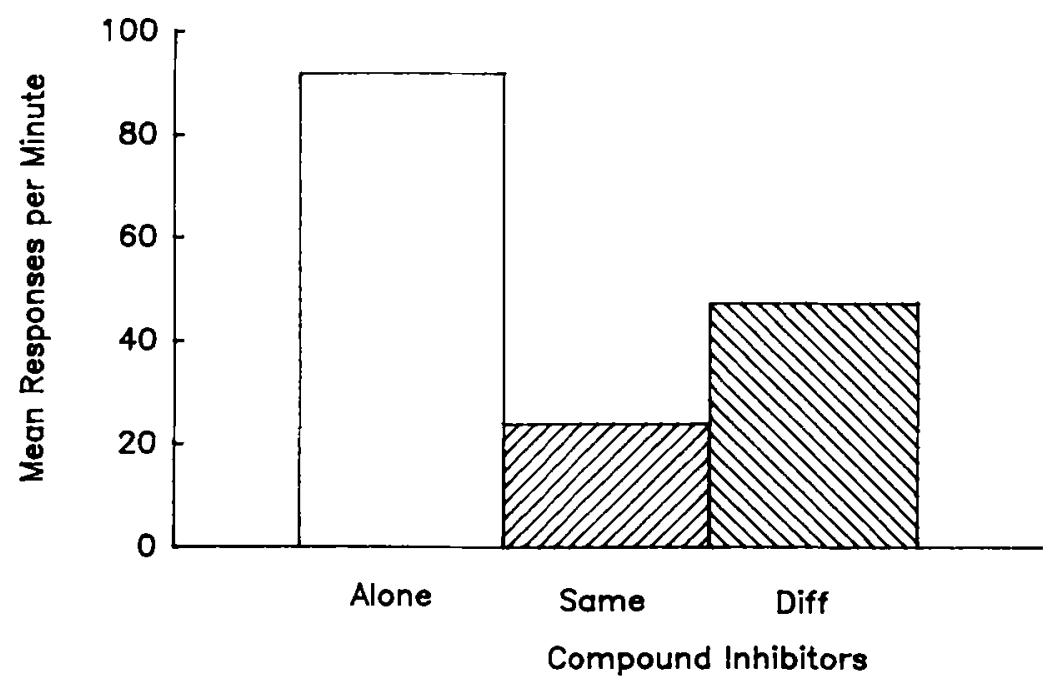

Figure 2. Transfer test in Experiment 1. Responding to the transfer keylight is shown when presented alone and when presented together with compounds of inhibitors originally trained with the same or different excitors. 
the presence of both compound inhibitors trained with the same target $[T(14)=0, p<.01]$ and different targets $[T(14)=1.5, p<.01]$. Although the present experiment contains no untrained comparison stimuli, those reductions agree with previous findings of successful transfer of inhibitors from one target to another in autoshaping. Of most interest, the compound inhibitor containing elements trained with the same target was more successful in reducing responding to $\mathrm{W}$ than was that containing elements trained with different targets $[T(12)=0, p<.01]$. That result suggests the involvement of the original training-target excitor in mediating transfer of inhibition. Although similar compounds were presented together with the transfer stimulus, they were differentially successful in producing transfer, depending on the identity of their training excitors.

The results of the second test confirmed these findings. During that test, the mean responses per minute were $101.6,57.2,35.6$, and 14.6, respectively, when the excitor was presented alone, when preceded by one of the inhibitory stimuli, when preceded by a combination of inhibitors based on different targets, and when preceded by a combination based on the same target. Presentation of either a single inhibitor or either of the two types of compound inhibitors reduced responding to the excitor relative to that observed when it was presented alone $[T \mathrm{~s}(14)<7, p \mathrm{~s}<.01]$. This suggests successful transfer to a new target by each of the individual inhibitors and by the compounds. Moreover, combinations of either same-target or different-target inhibitors reduced responding relative to that observed following presentation of a single inhibitor $\left[T_{\mathrm{s}}(15)<15, p \mathrm{~s}<.01\right]$. That is, summation of the effects of two inhibitors was observed whether those inhibitors had been trained with the same or a different target excitor. Finally, as in the first test, the combination of same-target inhibitors was more powerful in reducing responding to the excitor than was the combination of different-target inhibitors $[T(14)=$ $21, p<.05$ ].

These results suggest that although inhibitors transfer to new targets, they do so in such a way as to be sensitive to the identity of the original excitors with which they were trained.

\section{EXPERIMENT 2}

This experiment investigated the same question as did Experiment 1, but with a facilitation, rather than an inhibition, paradigm. Two different facilitators were trained as modulators for each of two excitatory targets. Then transfer to a new excitor was assessed for combinations of those facilitators that had been trained with either the same or different targets. The question of interest was whether facilitators would combine with differential success if they had been trained with the same and different original targets.

\section{Method}

Subjects and Apparatus. The subjects were 16 female White Carneaux pigeons maintained in the same manner as those of Experiment 1 . They had been used in prior autoshaping experiments with other stimuli and a different response key. Their treatments in the present experiment were random with regard to those prior treatments. The apparatus was that of Experiment 1.

Procedure. Because of their histories with autoshaping, no magazine training was necessary. Facilitation training began immediately and continued for 22 days. On each day, the birds received 12 nonreinforced presentations of each of the grids. In addition, they received 16 reinforced presentations of each grid, 8 of which were accompanied by an auditory stimulus and 8 of which were accompanied by one of the colors. For each bird, a particular auditory stimulus and color consistently accompanied a particular pattern, but the assignments were balanced across subjects. The auditory stimuli were each $15 \mathrm{sec}$ long, and the grid patterns occupied the final $5 \mathrm{sec}$. The color facilitators were each $5 \mathrm{sec}$ long and began $10 \mathrm{sec}$ prior to the 5-sec grids. Unlike in Experiment 1, a 5-sec gap was introduced between the visual modulator and the target. This was done to reduce the direct excitatory conditioning of the modulator that can occur in procedures in which compound stimuli terminate in the US. In prior experiments, these parameters have routinely been successful in producing facilitation without such excitatory conditioning (e.g., Rescorla, 1985).

Over the next 4 days, the white (W) stimulus was prepared as a transfer target. On each day, the birds received 365 -sec presentations of $W$. On the first 2 days, all trials were followed by $5 \mathrm{sec}$ of grain; on the next 2 days, all trials were nonreinforced. On the next day, the birds received a session of facilitation training identical to those given before.

On the next day, all birds received a test session. This session contained two nonreinforced presentations each of $W$ alone and accompanied by each of the four possible combinations of auditory and visual facilitators. On compound trials, the facilitators retained the durations and temporal relations to the target that had been used during training. A second test session, in which individual facilitators were also presented, was aborted because of an equipment failure.

\section{Results and Discussion}

Figure 3 shows the course of facilitation training, which was typical of that observed in this paradigm. Figure 3 combines responding over the identity of the stimuli and shows separately responding to the excitors (targets) when presented alone and when accompanied by either a visual or an auditory facilitator. After an initial rise in responding to the excitor on all trials, there was a gradual decline on the trials during which it occurred alone. At the end of training, responding was substantially less during the excitor alone than on trials containing either the auditory or the visual facilitator $[T \mathrm{~s}(15)=0, p s<.01]$.

The levels of responding did not differ on auditory- and visual-facilitator trials. However, one should be cautious in concluding that visual and auditory facilitators are equally successful. In paradigms of this sort, responding to the compounds is quite high and the discrimination appears as a loss in responding to the nonreinforced target alone. To the degree that a facilitator is ineffective, its target will remain high. However, since each target was used for both an auditory and a visual facilitator, should either of those facilitators be less effective, it would slow 


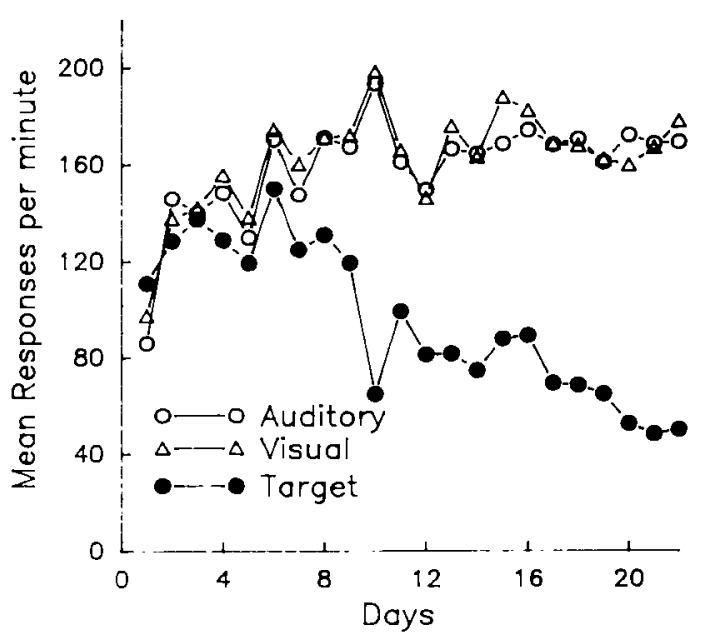

Figure 3. Responding to the target stimulus during acquisition of facilitation in Experiment 2. The keylight was reinforced when accompanied by either a visual or an auditory facilitator but not when presented alone (target).

the loss of responding to their shared target. That might make the facilitators appear equally effective when in fact they differ.

Figure 4 shows the results of most interest, those from the test session in which $W$ was presented alone and together with the compound facilitators. It is clear that the training and extinction of $\mathbf{W}$ had left it with a low level of responding when presented alone. However, responding during $\mathbf{W}$ was markedly elevated when presented together with a compound of facilitators trained with either the same $[T(12)=0, p<.01]$ or different targets $[T(11)$ $=0, p<.01]$. Although control comparisons were not included in this experiment, these results confirm earlier findings of substantial transfer of facilitation in this preparation to trained and extinguished targets.

Of most interest, the amount of facilitation was greater with the compound whose elements had been trained with the same original target $[T(14)=14, p<.02]$. That result suggests that the identity of the training target participates in mediating transfer of a facilitator to a new target.

\section{GENERAL DISCUSSION}

These results suggest that modulators that are trained with a common target better combine their effects in transferring to a new excitor. They implicate the original training target as a partial mediator in transfer of modulation.

The implications of these results are clearest for an interpretation of modulation in terms of simple action on the representation of the US. They suggest that a view that focuses only on the US, such as one in which modulators shift the threshold for US activation (e.g., Rescorla, 1979,1985 ), cannot give a complete account of modulation. Although such an account might survive earlier reports of incompleteness of transfer by appealing to generalization decrement, the present results present a much more serious challenge. Consequently, they join the findings on postacquisition manipulation of the original training target (Rescorla, 1991) in suggesting that the action of modulators must at least partially be directed at the training target.

But it is not clear in just what way the training target participates in transfer. One possibility is suggested by prior findings on within-compound associations (e.g., Rescorla, 1982). To the degree that each modulator acquires a within-compound association with its training target, presentation of that modulator might activate a representation of the target at the time of transfer. Rescorla (1982) attempted to explain incomplete transfer of inhi-

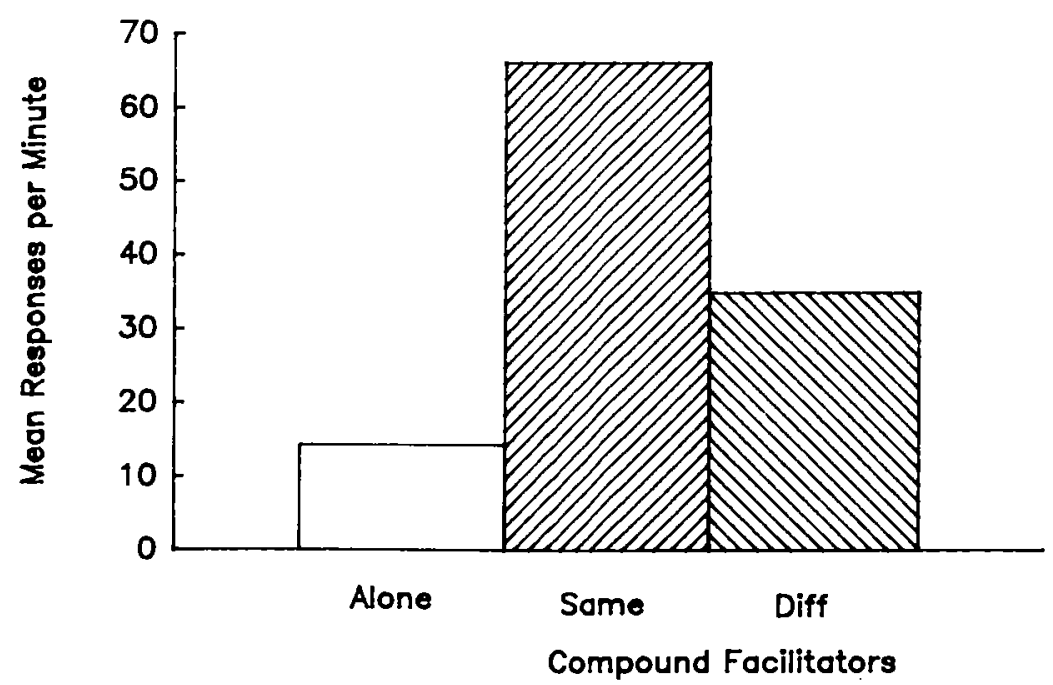

Figure 4. Transfer test in Experiment 2. Responding to the transfer keylight is shown when presented alone and when presented together with compounds of facilitators originally trained with the same or different targets. 
bition by suggesting that this importation of the original excitor into the transfer compound artificially augments the level of responding and masks the success of the transfer. One might make a similar argument when combining modulators associated with different excitors. The importation of two different targets might generate greater total excitation than would that of one target. Although this could be used to help understand the more successful combination of two inhibitors' sharing a target, it is less successful in explaining the results from facilitation paradigms. There, too, one would expect more total excitation from facilitators trained with different targets and hence more responding with such facilitators. However, the outcome of the present facilitation experiment is contrary to that prediction. Consequently, it does not appear possible to salvage a threshold-shifting view by combining it with within-compound associations that act in this manner.

Another possibility is that modulators act partly in a hierarchical fashion, activating a particular target A-US association (e.g., Holland, 1983). In such a view, transfer must then be accomplished by generalization between the $\mathrm{A}$ and $\mathrm{B}$ or between the A-US and B-US associations. Transfer would depend jointly on the similarity of $A$ to $B$ and the successful activation of the A-US association. It is possible that if two modulators are asked jointly to activate the same A-US association, they can do so with reasonable success, whereas when they are asked to activate different associations, the resultant competition diminishes the activation of either. Of course, one could envision such a process functioning so as to make either same or different target modulators the more successful. As in other attempts to use logic of this sort (e.g., Rescorla, 1990; Rescorla \& Furrow, 1977), either differential outcome implies the encoding of the target identity.

Although the present results may be taken as evidence for a hierarchical action of modulators, they do not support an interpretation in terms of modulators and targets being encoded in a different memory system (e.g., Holland, $1989 \mathrm{c})$. According to such a view, the transfer of a modulator to a novel target depends upon that target's having also been involved in a modulatory training paradigm. However, with the present procedures, transfer was routinely observed to targets that had experienced only simple Pavlovian training and extinction.

Finally, results like those found here do little to encourage a view of facilitation, inhibition, and transfer in terms of perceptual configurations. For instance, Pearce (1987) has recently suggested that it is profitable to view discriminations of the sort studied here as differentially reinforcing configural stimuli that differentially generalize to each other and to transfer presentations. According to that view, a conditioned-inhibition procedure of the type conducted here involves reinforcing two excitors, $X$ and $Y$, but nonreinforcing four configurations that might be described as AX, BX, CY, and DY. The various transfer test compounds involving a new target, $Z$, would then be described as $A B Z$ and $C D Z$ for the inhibitors trained with a common target but as $\mathrm{ADZ}$ and $\mathrm{CBZ}$ for the inhib- itors trained with different targets. Responding would be governed by the similarity of each test configuration to the various training configurations. But there is no reason to believe that the compounds containing two modulators trained with the same target, $\mathrm{ABZ}$ and $\mathrm{CDZ}$, are more similar to the various training compounds than are those containing modulators trained with different targets, ADZ and CBZ. In agreement with that argument, a simulation of these procedures with the model described by Pearce (1987) fails to yield the results observed here.

Consequently, these results suggest an involvement of the original training target when a modulator transfers to a new target. Moreover, that involvement goes beyond that anticipated on the basis of perceptual interactions among targets and modulators. That result creates some difficulty for each of the available accounts of modulation.

\section{REFERENCES}

Holland, P. C. (1983). “Occasion-setting”' in Pavlovian feature positive discriminations. In M. L. Commons, R. J. Hermstein, \& A. R. Wagner (Eds.), Quantitative analyses of behavior: Vol. IV. Discrimination processes (pp. 183-206). Cambridge, MA: Ballinger.

Holland, P. C. (1985). The nature of conditioned inhibition in serial and simultaneous feature negative discriminations. In R. R. Miller \& N. E. Spear (Eds.), Information processing in animals: Conditioned inhibition (pp. 267-297). Hillsdale, NJ: Erlbaum.

Holland, P. C. (1989a). Acquisition and transfer of conditioned discrimination performance. Joumal of Experimental Psychology: Animal Behavior Processes, 15, 154-165.

Holland, P. C. (1989b). Feature extinction enhances transfer of occasion setting. Animal Learning \& Behavior, 17, 269-279.

HollaNd, P. C. (1989c). Transfer of negative occasion setting and conditioned inhibition across conditioned and unconditioned stimuli. Journal of Experimental Psychology: Animal Behavior Processes, 15, 311-328.

Pearce, J. M. (1987). A model of stimulus generalization for Pavlovian conditioning. Psychological Review, 94, 61-73.

Rescorla, R. A. (1979). Conditioned inhibition and extinction. In A. Dickinson \& R. A. Boakes (Eds.), Mechanisms of learming and motivation: A memorial volume to Jerzy Konorski (pp. 83-110). Hillsdale, NJ: Erlbaum.

Rescorla, R. A. (1982). Some consequences of associations between the excitor and the inhibitor in a conditioned inhibition paradigm. Journal of Experimental Psychology: Animal Behavior Processes, 8, 288-298.

Rescorla, R. A. (1985). Inhibition and facilitation. In R. R. Miller \& N. E. Spear (Eds.), Information processing in animals: Conditioned inhibition (pp. 299-326). Hillsdale, NJ: Erlbaum.

Rescorla, R. A. (1989). Simultaneous and sequential conditioned inhibition in autoshaping. Quarterly Journal of Experimental Psychology, 41B, 275-286.

Rescorla, R. A. (1990). Evidence for an association between the discriminative stimulus and the response-outcome association in instrumental leaming. Joumal of Experimental Psychology: Animal Behavior Processes, 16, 326-334.

Rescorla, R. A. (1991). Transfer of inhibition and facilitation mediated by the original target stimulus. Animal Learning \& Behavior, 19, 65-70.

Rescorla, R. A., \& FURrow, D. R. (1977). Stimulus similarity as a determinant of Pavlovian conditioning. Journal of Experimental Psychology: Animal Behavior Processes, 3, 203-215

(Manuscript received February 22, 1991; revison accepted for publication July 11, 1991.) 